19 Revue d'histoire du XIXe siècle

Société d'histoire de la révolution de 1848 et des

révolutions du XIXe siècle

39 | 2009

Le monde de l'imprimé: des territoires aux acteurs -

Education et politique - Histoires politiques

\title{
La légitimité incertaine (1814-1853) : retour sur les faux Louis XVII
}

Uncertain legitimacy (1814-1853: re-reading the false Louis XVII

Paul Airiau

\section{OpenEdition}

Journals

Édition électronique

URL : http://journals.openedition.org/rh19/4142

DOl : $10.4000 /$ rh 19.4142

ISSN : $1777-5329$

Éditeur

La Société de 1848

Édition imprimée

Date de publication : 10 décembre 2009

Pagination : 115-127

ISSN : 1265-1354

\section{Référence électronique}

Paul Airiau, "La légitimité incertaine (1814-1853) : retour sur les faux Louis XVII », Revue d'histoire du XIXe siècle [En ligne], 39 | 2009, mis en ligne le 15 décembre 2012, consulté le 03 mai 2019. URL :

http://journals.openedition.org/rh19/4142 ; DOI : 10.4000/rh19.4142 


\section{PAUL AIRIAU}

\section{La légitimité incertaine (1814-1853): retour sur les faux Louis $X V I I^{1}$}

Qu'un prétendu Louis XVII soit réellement le fils de Louis XVI importe peu à l'historien ${ }^{2}$. En revanche, il lui appartient pleinement de se pencher sur la signification des aventures des faux dauphins après 1815. Certes, le «survivantisme» (la croyance en la survie de Louis XVII, évadé du Temple) fut et demeure une réalité marginale, malgré des succès passagers. Mais que des ultras, après 1815, en arrivent à accuser Louis XVIII puis Charles X d'avoir usurpé le trône de leur neveu en dit long sur l'incertitude de ceux qui apparaissent alors comme les soutiens les plus évidents de la monarchie restaurée. Ils semblent de ce fait moins éloignés qu'on ne pourrait le penser de tous ceux qui, royalistes, bonapartistes ou républicains, contestent les fondements de l'exercice du pouvoir par les Bourbons.

Ainsi voudrait-on montrer le rôle révélateur, autant que catalyseur, du survivantisme. Son apparition précoce et sa permanence renouvelée entre 1815 et la Seconde République - le baron de Richemont, le dernier grand prétendant, meurt en 1853 - soulignent en particulier la réitération incessante d'interrogations sur la légitimité des détenteurs du pouvoir politique ${ }^{3}$.

1. Outre Lionel Parois, Essai de bibliographie sur Louis XVII, Paris, 1992 (nombreuses publications depuis), sont indispensables : Joseph-Marie Quérard, Les supercheries littéraires dévoilées. Galerie des auteurs apocryphes, supposés, déguisés, plagiaires, et des éditeurs infidèles de la littérature française pendant les quatre derniers siècles: Ensemble les industriels littéraires et les lettrés qui se sont anoblis à notre époque, Paris, chez l'éditeur, 5 tomes, tome 3, 1850, p. 17-121; Léon de la Sicotière, "Les faux Louis XVII (première partie)", "Les faux Louis XVII (seconde partie)", Revue des questions historiques, volume 32, $1^{\text {er }}$ juillet 1882, p. 147-209, $1^{\text {er }}$ octobre 1882, p. $494-591$ (Les faux Louis XVII, Paris, V. Palmé, 1882); René Le Conte, Les faux Dauphins, Paris, PUF, 1924; Jacques Hamann, Maurice Étienne, Louis XVII et les 101 prétendants, Paris, Le Sémaphore, 1999.

2. Sauf Yves-Marie Bercé, Le roi caché. Sauveurs et imposteurs. Mythes politiques dans l'Europe moderne, Nouvelles Études historiques, Paris, Fayard, 1990, p. 328-339, qui inscrit les faux dauphins dans la longue durée et apporte des grilles d'analyse (p. 378-415).

3. Philippe Boutry, "Carisma di crisi e crisi del carisma : Luigi XVIII e il profeta Martin", dans JeanMichel Sallmann, Philippe Levillain [dir.], Forme di potere e pratica del carismo, Napoli, Liguori editore, 1984, p. 93-108; Mona Ozouf, "L'idée et l'image du régicide dans la pensée contre-révolutionnaire : l'originalité de Ballanche", dans François Lebrun, Roger Dupuy [dir.], Les résistances à la Révolution. Actes du colloque de Rennes (17-21 septembre 1985), Paris, Imago, 1987, p. 331-341; Emmanuel de Waresquiel, L'histoire à rebrousse-poil. Les élites, la Restauration, la Révolution, Paris, Fayard, 2005, p. 22-24 et 43-68, reprenant et approfondissant Emmanuel de Waresquiel, "Talleyrand et la légitimité : la "révolution” du 31 mars 1814 ", dans Jean-Yves Mollier, Martine Reid, Jean-Claude Yon [dir.], 
Sont-ils fondés à l'exercer non seulement légalement (car la légalité peut toujours être manipulée) mais aussi au nom d'une vérité qui fonderait un droit? Sont-ils les instruments de Dieu, les justes bénéficiaires d'une règle ancestrale, l'expression de la raison, de la volonté nationale, de la délégation populaire, les précurseurs de l'avenir? Tous ces points ne pouvant être abordés ici, on se concentrera sur un seul : la multiplication des faux Louis XVII traduit une insatisfaction politique et le remodelage des notions cardinales pour la légitimité royale que sont la succession dynastique par primogéniture masculine, l'articulation du corps politique et du corps physique du roi.

\section{Multiplication des Louis XVII et insatisfaction politique}

La dissociation entre la souveraineté et la légitimité et entre le roi et la nation, opérée à partir de 1789 , n'a jamais été vraiment surmontée malgré les multiples tentatives monarchiques, républicaines et impériales. L'ouverture partielle puis croissante du champ des possibles à partir de 1812-1813 profite finalement à Louis XVIII, qui put et sut s'imposer face à Napoléon, aux combinaisons de Talleyrand et du Sénat, aux Alliés mal accordés, aux Français, à ses propres partisans. Charles $\mathrm{X}$ hérita de son frère, mais sans la légitimité proprement personnelle construite par celui-ci. Louis-Philippe s’imposa et fut imposé par les élites politiques contre son cousin, son neveu, les républicains et le peuple parisien. Tous trois alimentèrent leur légitimité, chacun à sa manière, par l'exercice du pouvoir, la mise en scène royale, le remodelage de la sacralité, l'exploitation de l'enracinement historique, les projets idéologiques, l'utilisation de la Charte ${ }^{4}$.

Repenser la Restauration. Actes du colloque, Paris, Musée de la vie romantique, Châtenay-Malabry, Maison de Chateaubriand, septembre 2003, organisé par le Centre d'histoire culturelle des sociétés contemporaines, Paris, Nouveau monde, 2005, p. 57-68; Sheryl Kroen, Politics and Theater. The Crisis of Legitimacy in Restoration France, 1815-1830, Berkeley, Los Angeles, London, University of California Press, 2000, spécialement p. 35-36; Emmanuel Fureix, La France des larmes. Deuils politiques à l'âge romantique (1814-1840), Seyssel, Champ Vallon, 2009; François Guizot et la culture politique de son temps, textes rassemblés et présentés par Marina Valensise, Paris, Gallimard/Le Seuil, 1991; Pierre Rosanvallon, Le moment Guizot, Paris, Gallimard, 1985.

4. Emmanuel de Waresquiel, «Portraits du roi et de ses élites sous la Restauration et la Monarchie de Juillet, une contribution à l'étude des représentations du pouvoir ", Versalia. Revue de la Société des amis de Versailles, $n^{\circ}$ 9, 2006, p. 178-194. Sur la Restauration : Volker Sellin, "Restauration et légitimité en 1814», Francia, 26/2, 1999, p. 115-129; Martin Wrede, "Le portrait du roi restauré, ou la fabrication de Louis XVIII", Revue d'histoire moderne et contemporaine, tome 53, ${ }^{\circ}$ 2, avril-juin 2006, p. 112-138; Pascal Simonetti, "Mourir comme un Bourbon : Louis XVIII, 1824", Revue d'histoire moderne et contemporaine, tome 42, ${ }^{\circ}$ 1, janvier-mars 1995, p. 91-106; Emmanuel Fureix, La France des larmes..., ouv. cité, p. 26-34, 97-109, 170-183 et 219-317. Sur Louis-Philippe : Jo Burr Mardagant, 'Gender, vice and the political imaginary in postrrevolutionary France: reinterpreting the failure of the July Monarchy', The American Historical Review, volume 104, n 5, décembre 1999, p. 1460-1496, et 'Representing Queen Marie-Amélie in a "bourgeois" monarchy', Historical Reflections, volume 32, n 2, 2006, p. 421-451; Bradford Brown, 'The rhetoric of premature mourning. Assassination attempts and the civil religion of the July Monarchy', Proceedings of the Western Society for French History, volume 29, 2001, p. 80-88; Pierre Karila-Cohen, "Charles de Rémusat et l'impossible reconstruction de la Monarchie de Juillet", Revue d'Histoire moderne et contemporaine, tome 44, n 3, juillet-septembre 1997, 
Mais Louis XVII n'avait pas disparu de la scène politique. Les proclamations de Louis XVIII atteignirent sans doute davantage les élites que le peuple. Des Français en étaient restés au fils de Louis XVI, malgré l'annonce de sa mort en 1795. Fin 1813-début 1814, les révoltes de conscrits d'une partie de la Flandre française, rapidement devenues soulèvement rural antinapoléonien, se firent au nom de Louis XVII (sobriquet qui resta à leur chef) $)^{5}$. L'arrivée sur le trône de Louis XVIII n'empêcha même pas la multiplication des figures de son neveu'.

Mathurin Bruneau est le premier cas significatif. Son aventure commence fin 1815 et s'achève en février 1818 par un procès le condamnant à sept ans de prison (dont deux pour outrage au tribunal), où il meurt en 1822 . Laffaire est suffisamment prise au sérieux pour que des contre-feux soient ouverts et que Béranger en fasse une chanson ( «Le prince de Navarre ou Mathurin Bruneau", qui lui vaut un procès) ${ }^{7}$. Louis XVII devient alors un personnage que l'on investit. Sept hommes s'identifient publiquement à lui entre 1817 et 1824, sans compter les rumeurs (en septembre 1823, près d'Angers, un homme raconte que la duchesse d'Angoulême a un frère vivant dans «l'isle de Maroc») ${ }^{8}$. Sous la monarchie de Juillet, deux prétendants dominent. Le baron de Richemont apparaît en 1831. Condamné en 1834, évadé, il poursuit sa carrière jusqu’à son décès en 1853. Karl-Wilhelm Naundorff surgit en 1833. Expulsé en Angleterre en 1836, il maintient ses prétentions jusqu'à sa mort à Delft en $1845^{9}$.

Cette multiplication des faux Louis XVII a immédiatement fait l'objet d'une lecture psychiatrique, contribuant à enrichir la figure du mythomane ${ }^{10}$. On peut également délaisser l'histoire singulière des prétendants pour s'attacher à la signification sociale de leur revendication. Le destin du jeune roi sous la Restauration peut en rendre compte. En effet, le régime a involontairement alimenté l'incertitude sur sa mort. La loi du 19 janvier 1816 instituant l'expiation de la mort de Louis XVI prévoit l'érection de monu-

p. 404-423; Michaël Marrinan, Painting Politics for Louis-Philippe. Art and Ideology in Orleanist France, New Haven, Yale University Press, 1988.

5. "Affaire du prétendu Louis XVII", L'ami de la religion et du roi, 04/11/1834, p. 32; Paul Fauchille, Une chouannerie flamande au temps de l'Empire (1813-1814). Louis Fruchart, dit Louis XVII, d'après des documents inédits, Paris, A. Pedone, 1905.

6. Sans compter un faux Bonaparte, Jean Charnay, annonçant l'invasion de la France par des «Turcs et des barbaresques", jugé à Trévoux en septembre 1817, condamné à cinq ans de prison : Bénaben, "Politique», Mercure de France, 20 septembre 1817, p. 573.

7. Pour les publications défendant la mort de Louis XVII, voir en particulier Joseph-Marie Quérard, Les supercheries..., ouv. cité; J. [eanne] de Saint-Léger, Était-ce Louis XVII évadé du Temple?, Documents inédits tirés des Archives de Police et des Greffes judiciaires, Paris, Librairie Perrin et Cie, 1911.

8. Arch. Nat. (Archives nationales), BB30 198 (procureur général du roi près la cour royale d'Angers au secrétaire général du ministère de la Justice, 24/09/1823). Sur les prétendants, voir les références de la note 1 .

9. En sus des ouvrages de la note 1, Edmond Dupland, Naundorff limposteur, Paris, Olivier Orban, 1990.

10. T. Haustgen, M.-L. Bourgeois, «L'évolution du concept de mythomanie dans l'histoire de la psychiatrie», Annales Médico-psychologiques. Revue Psychiatrique, volume 165, n 5, juin 2007, p. 334-344. 
ments à Marie-Antoinette, Madame Élisabeth et Louis XVII, intégré grâce à l'intervention de Chateaubriand à la Chambre des pairs le 9 janvier. Une ordonnance du 14 février 1816 prévoit d'achever la Madeleine pour les y placer - ce qui ne se réalisa jamais, l'église n'étant pas terminée. Si les corps de Louis XVI et de sa femme sont identifiés et inhumés à Saint-Denis, celui de Louis XVII n'est pas retrouvé lors des fouilles dans le cimetière de Sainte-Marguerite de mars à juin 1816 (il en va de même pour sa tante). Les négociations avec le médecin Pelletan pour récupérer son cœur (dérobé lors de l'autopsie en 1795) n'aboutissent pas ${ }^{11}$. Le transfert prévu à SaintDenis ne se fait donc pas. Le jeune roi, intégré par la bande au culte de la famille royale et de la dynastie, est en plus peu représenté pour lui-même. Sur les 26 estampes qui le figurent, 23 sont éditées entre 1814 et 1816 (dont 19 en 1814, la dernière en 1826). Il n'y est seul que dix fois, étant souvent associé à ses parents ou son père (sept fois), à sa famille proche ou élargie (jusqu'à des victimes de la Révolution), à sa dynastie ou à des souverains vivants. Son statut est enfin incertain. En témoignent les appellations telles "Louis XVII» (huit occurences), «dauphin» (dix occurences), «LouisCharles» (sept occurences), «fils de Louis XVI» (deux occurences), qui sont parfois associées dans un même texte ${ }^{12}$.

Cet effacement peut expliquer qu'une foule essaye d'apercevoir Bruneau lors de son arrestation à Saint-Malo et de son transfert à Rennes en décembre 1815, et les voyages ensuite entrepris par certains depuis Paris jusqu'à Rouen pour le visiter. Mais surtout, l'attitude gouvernementale paraît ambiguë. En prévision du procès, le procureur de Rouen demande à pouvoir prouver l'usurpation d'identité. Mais Decazes refuse toute publication des actes établissant la mort de Louis XVII. Une brochure saisie par la police, Les pourquoi de 1817, rédigée sans doute par le publiciste Gabriel BourbonLeblanc, s'interrogea sur le refus de confronter Bruneau à des personnes ayant connu le roi. Durant l'audience, l'avocat du prétendant, qui s'était engagé à ne pas aborder l'éventuelle évasion du Temple, l'évoque malgré tout. Il est immédiatement censuré par le président du tribunal. Enfin, la visite à Rouen de deux gardes du corps de Monsieur, sans doute mandés par la duchesse d'Angoulême, participe à l'interrogation : douterait-elle de la mort de son frère? ${ }^{13}$ Ainsi, le choix politique de la judiciarisation, s'il affirme la mort contre la survie, publicise la question et rend politiquement disponible Louis XVII.

11. Emmanuel Fureix, La France des larmes..., ouv. cité, p. 151-167, 197-209; Philippe Delorme, Louis XVII, la vérité. Sa mort au Temple confirmée par la science, Paris, Pygmalion/Gérard Watelet, 2000, contesté par Philippe Alexandre Boiry, Louis XVII avait-il deux cours?, Versailles, Éditions de Paris, 2004.

12. Décompte à partir de 'The Image of France 1793-1880 at ARTFL' (http://www.lib.uchicago.edu/efts/ ARTFL/projects/mckee/), utilisant les estampes parues dans la Bibliographie de la France.

13. J. [eanne] de Saint-Léger, Était-ce..., ouv. cité, p. 25, 35, 77-78, 86-88, 209 et 232-234. Voir aussi

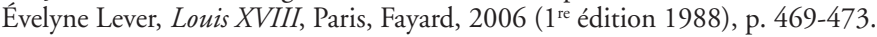


Il sert alors à la contestation. Les proclamations affichées à Rouen en son nom en 1817 faisaient jouer la crise frumentaire (le pain bon marché), le «bon roi " (référence à Henri IV, le roi nourricier, réactivée par Louis XVIII et utilisée ici pour le contester), le patriotisme (référence à la mise en congé des demi-soldes). Bruneau attire pourtant plus de royalistes que d'anti-Bourbons : Françoise Desprez, qui se présente comme combattante vendéenne, le soutient puis se rétracte partiellement pour se contenter d'affirmer que Louis XVII avait été enlevé par les Vendéens; un comte de Lantivy, ancien chouan (sans doute Jacques de Lantivy de Kervéno), diffuse ses positions en Angleterre; Nicolas-Philibert Le Chandelier de Pierreville, chouan mayennais, envisage de s'installer près de Rouen pour aider à son évasion; le lieutenant-colonel Jacques-Charles de Foulques, ancien émigré, est son messager auprès de la duchesse d'Angoulême ${ }^{14}$. Parfaitement inscrite dans le cadre de la bouffée de rumeurs de 1814-1817, l'affaire Bruneau fut sans doute exploitée contre le gouvernement, même par des royalistes, au risque de saper la légitimité de Louis XVIII. On peut alors y voir une désillusion envers le roi dépassant la récrimination ultra ${ }^{15}$.

Le faux dauphin permet donc d'exprimer indirectement son hostilité au régime. Le sous-préfet de Fougères, visant clairement républicains et bonapartistes, écrit ainsi en 1815 à propos de Bruneau : «[...] les gens les moins tendres pour l'auguste famille qui nous gouverne, se sont fort attendris sur le sort de cet infortuné jeune homme, s'il est réellement celui qu'on croit mort ${ }^{16}$. En 1824 , certains républicains exilés à Londres accueillent même avec une certaine faveur la revendication de Victor Persat qui vient de se déclarer Louis XVII aux États-Unis. S'il est vrai que son frère Maurice est des leurs, ils s'amusent pourtant à imaginer un retour dans une France libérale où règnerait un Victor qui leur octroierait des postes. Cette bouffonnerie leur permet de rêver au succès républicain et de rejeter une monarchie restaurée convoitant le "pouvoir absolu» et ne respectant pas le "gouvernement représentatif $»^{17}$. En somme, dans ce cas précis, l'image

14. Mémoires de Billard de Veaux (Alexandre), ancien chef vendéen ou Biographie des personnes marquantes de la Chouannerie et de la Vendée pour servir à l'histoire de France et détourner les habitants de l'Ouest de toute tentative d'insurrection, Paris, Lecointe et Pougin, Versailles, Borreswil, 3 tomes, tome 2, 1832, p. 262-263. J.[eanne] de Saint-Léger, Était-ce..., ouv. cité, p. 86-87, 148; René Le Conte, Les faux dauphins, ouv. cité, p. 62-65.

15. François Ploux, De bouche à oreille. Naissance et propagation des rumeurs dans la France du XIXe siècle, Collection historique, Paris, Aubier, 2003, p. 130-170. Sur le cas Bruneau, Yann Guérin, «Le Dauphin de 1815 : étude d'une rumeur", Annales de Bretagne et des pays de l'Ouest, volume 110, n 2, 2003, p. 111-128.

16. J.[eanne] de Saint-Léger, Était-ce..., ouv. cité, p. 15.

17. Frédéric Degeorge, "Les proscrits de la Restauration", dans Paris révolutionnaire, Paris, Pagnerre, 1848 , p. 186-190. Voir aussi la mise en cause du gouvernement et de la Cour dans la notice «BourbonBusset dit Leblanc (Gabriel)» de la Galerie historique des contemporains, ou nouvelle biographie, nouvelle édition, dans laquelle se trouvent réunis les hommes morts ou vivants, de toutes les nations, qui se sont fait remarquer à la fin du $18^{e}$ siècle et au commencement de celui-ci, par leurs écrits, leurs actions, leurs talents, leurs vertus ou leurs crimes, seconde édition, Bruxelles, Aug. Wahlen et Comp., 10 volumes, volume 2, 1822, p. 363-364. 
du roi populaire, simple et bon vivant (selon Maurice, Victor couronné se contenterait d'alcool et de femmes...), remodelage d'Henri IV que Louis XVIII est incapable de remplacer, n'a pas totalement disparu des esprits même républicains. Le monarque peut ici demeurer une référence positive, sous des traits qui permettent de l'opposer au roi régnant.

\section{Primogéniture et dynastie}

Pendant la monarchie de Juillet d'autres éléments se font jour. Naundorff fut reconnu comme Louis XVII par deux générations distinctes de royalistes. D'abord des personnes ayant connu la famille royale : Agathe de Rambaud (berceuse du dauphin), Étienne de Joly (éphémère ministre de la Justice notamment lors du 10 août 1792), Denis Marco, ancien huissier de madame Victoire; et des émigrés, des combattants royalistes et des ultras d'une certaine importance : Sosthène de La Rochefoucauld (reconnaissance cependant incertaine et finalement abandonnée), le comte Alexis de Lancran de Bréon (combattant de Coblence, maréchal de camp, réprimant le soulèvement républicain du général Berton en 1822), le marquis Louis Le Gendre de La Ferrière (combattant dans l'armée royaliste de l'Ouest), Alexandre de Cosson (émigré de Coblence). Puis des fonctionnaires ou de simples particuliers trop jeunes pour avoir connu la Révolution : François Albouys (juge à Cahors, démissionnaire en 1830), Modeste Gruau (procureur de Mayenne, épuré en 1830), Fernand Geoffroy (secrétaire du vice-gouverneur des pages de Charles X, émigré de l'intérieur en 1830), Édouard Bricon (libraire et éditeur), les frères Laprade (Jean-Baptiste, prêtre, et Xavier, avocat). Chez les richemontistes, on peut relever Amédée Nicolas (militaire démissionnaire en 1830, devenu avocat) et la comtesse de Vabres d'Apchier (dont le père et un frère furent exécutés comme "fédéralistes" à Lyon en 1793, et le mari pressenti comme député en 1824) ${ }^{18}$.

Ces royalistes devenus légitimistes s'éloignent pourtant de «l'enfant du miracle». D'importantes déceptions peuvent l'expliquer. Ainsi, Agathe de Rambaud, issue d'une famille libérale de l'administration de la Marine avant 1789, est peu récompensée en 1815 de sa fidélité au dauphin, maintenue jusqu'au 10 août 1792. Elle n'obtient qu'une pension de 1000 francs et a du mal à assurer une situation à son fils, ancien fonctionnaire impérial qui a suivi le roi à Gand. Cosson, installé à partir de 1797 en Angleterre où il se marie en 1810, fait une tentative infructueuse de retour en France après

18. Voir notamment Michel Denis, Les royalistes de la Mayenne et le monde moderne (XIXé-XXe siècles), Paris, Kincksieck, 1977, p. 215-216; Guy de Rambaud, Pour l'amour du dauphin. Madame Agathe de Rambaud, Versailles, 10 décembre 1764, Aramon, 18 octobre 1853, Parçay-sur-Seine, Anovi, 2005; notices sur naundorffistes et vintrasiens dans Jean-Marie Mayeur et Yves-Marie Hilaire [dir.], Dictionnaire du monde religieux dans la France contemporaine, 10 tomes, tome 10, Jean-Pierre Chantin [dir.], Les marges du christianisme. "Sectes", dissidences, ésotérisme, Paris, Beauchesne, 2001. 
1815. Les prétendants surent aussi être convaincants, et profitèrent de l'influence d'un prophétisme politique plus ou moins manipulé (on le verra plus loin). Mais, pour être efficaces, ces facteurs supposent un terrain favorable. Il faut donc s'interroger sur le rapport à la légitimité de ces survivantistes. Dans le cas de la Rochefoucauld, ses Mémoires justifient longuement, à partir de ce point, que de grands ultras aient cru en la survie de Louis XVII :

«[...] en ce qui touche les amis des Bourbons, plus ils étaient sérieusement attachés au principe de la légitimité, plus aussi ils devaient apporter de soins à rechercher et à constater la vérité sur un point si important de politique et de fidélité. [...] Il n'était donc ni rare ni surprenant de voir quelques personnes des plus graves et des plus honorables, ajouter une croyance empressée, bien que circonspecte, douteuse et momentanée, aux prétentions de ceux qui se disaient Louis XVII et aux témoignages qu'ils en donnaient. [...] tout voir, tout écouter, tout apprécier en pareille matière, n'était-ce pas le meilleur moyen de déconcerter la malveillance, de confondre la calomnie, de déjouer l'intrigue, de reconnaître l'erreur ou, qui sait, la vérité peutêtre?" ${ }^{19}$

Ainsi, l'incertitude sur la mort de Louis XVII et le souci de la légitimité conduisent à remettre en cause implicitement, avec gêne mais réellement, la légitimité des rois trônant. Cela pourrait n'exprimer qu'une insatisfaction politique profonde, comme celle de ces ultras ou de ces chouans qui envisagèrent le remplacement de Louis XVIII par Monsieur ${ }^{20}$. Mais c'est en fait bien une profonde incertitude sur la légitimité du pouvoir qui se manifeste. Car ce n'est plus seulement, comme chez les royalistes déçus par Louis XVI entre 1789 et 1792, une interrogation sur l'exercice réel du pouvoir et l'appel à la fidélité à ce qu'il implique; ou une contestation de la nationalisation de la royauté et de la «royalisation" de la nation. Ici, la fidélité au principe même de la succession par ordre de primogéniture mâle l'emporte sur la fidélité à la personne du roi régnant. L'exercice légitime du pouvoir est, dans le cadre dynastique, rendu strictement dépendant d'une des règles non écrites de la monarchie d'avant $1789^{21}$. Cette loi fondamentale est jugée plus importante que la présence sur le trône d'un frère de Louis XVI, même s'il défend une royauté contre-révolutionnaire (dès avant 1830 , de hauts responsables ecclésiastiques sont convaincus de l'illégitimité de Charles X, malgré le projet ultra de ce dernier ${ }^{22}$ ). Quand bien même elle serait un moindre mal, elle est foncièrement illégitime car voilant les "attributs de la couronne». Le sang s'impose donc contre le sang, la primogéniture contre la dynastie, au point,

19. Mémoires de Monsieur le Vicomte de la Rochefoucauld, aide de camp du feu roi Charles X, Paris, Allardin, 5 tomes, tome 5, 1837, p. 46-47.

20. Michel Denis, Les royalistes..., ouv. cité, p. 123-124, 171.

21. Jean Barbey, Etre roi. Le roi et son gouvernement en France de Clovis à Louis XVI, Paris, Fayard, 1992, p. $17-70$ (surtout $37-42$ ).

22. René-François Rohrbacher, Histoire universelle de l'Église catholique, Paris, Gaume Frères, 1848, 29 tomes, tome 28, p. 291-292. 
chez certains ultras, de miner la légitimité du roi effectivement régnant, et quel que puissent être les conséquences. Car affirmer la survie de Louis XVII est en fait affirmer une usurpation, volontaire ou non, destructrice de toute légitimité, créatrice d'une radicale incertitude politique et susceptible de remettre en cause la Restauration et la dynastie bourbonnienne. De telles conséquences expliquent peut-être que le survivantisme ne puisse se manifester réellement qu'après 1830 : il fallait qu'il fût inappliquable pour pouvoir se développer.

Bien sûr, cela suppose de tenir pour vraie la survie de Louis XVII. La position survivantiste ne serait qu'une variante de l'application logique des règles successorales. Cependant, il faut ici prendre en compte la modification de la conception dynastique issue de la Révolution. Si la succession par primogéniture masculine n'est jamais oubliée, la succession simplement dynastique, sur des critères entrecroisant idéologie et considérations de personne, s'est vulgarisée : projet de substitution du comte de Provence à Louis XVI en 17891790 , projets d'accès au trône de la branche d'Orléans, organisation de la dynastie napoléonienne, projets ultras de renversement de Louis XVIII23. La définition comme "provocation au crime", par la loi Serre du 17 mai 1819, de «l'attaque formelle contre [...] l'ordre de succession au trône», laisse aussi deviner que cette question inquiète le pouvoir. N'évolue-t-il d'ailleurs pas lui-même, afin de préserver la dynastie? Si Charles X refuse le décret de Ferdinand VII abolissant la loi salique en Espagne (29 mars 1830), il abdique pourtant en faveur du duc de Bordeaux, en imposant un acte identique à son fils. Le monde légitimiste se déchirera sur cet acte jusqu'à la mort du duc d'Angoulême ${ }^{24}$.

Les survivantistes montrent ainsi que le principe dynastique est en luimême atteint, puisque ses conséquences pratiques iraient contre son objectif (garantir la continuité de l'exercice du pouvoir), et que sa fragilisation empêche de le faire fonctionner comme avant 1789. À ce titre, ils sont aussi révélateurs que l'indéniable échec pratique de la succession dynastique en France au XIXe siècle. Même si tous les monarques s'inscrivent dans une dynastie (qu'ils la prolongent ou la fondent) et tentent de renouer ce qui s'était brisé entre 1789 et 1793, l'union d'une famille et de la France, la nation fraternelle, lentement, progressivement, remplace désormais la paternité royale ${ }^{25}$.

23. Sur l'affaire Favras, voir Évelyne Lever, Louis XVIII, ouv. cité, p. 133-148.

24. Hugues de Changy, Le mouvement légitimiste sous la monarchie de Juillet (1833-1848), Rennes, Presses Universitaires de Rennes, 2004, p. 61-63, 74-75, 144-145 et 177-179; Stéphane Rials, Le légitimisme, Que sais-je?, Paris, PUF, 1983, p. 15-17.

25. Lynn Hunt, The Family Romance of the French Revolution, Berkeley, University of California Press, 1992, traduction française Le roman familial de la Révolution française, Paris, Albin Michel, 1995. 


\section{IDÉOLOGISATION DE LA LÉGITIMITÉ ET CORPS DU ROI}

Ces survivantistes représentent alors une autre conception royaliste de la légitimité, distincte des partisans du droit historique ou des tenants du droit divin $^{26}$. Ils réduisent la légitimité au strict respect d'une procédure, à un formalisme juridique. Ce formalisme est cependant articulé à une idéologisation de la légitimité qui peut les rapprocher du droit divin. En effet, les survivantistes font aussi dépendre la légitimité du respect de la volonté divine, par le biais du prophétisme. La contestation de Louis XVIII, puis partiellement de Charles X, s'alimente ainsi dans une partie du monde ultra aux prophéties politiques circulant de manière manuscrite ou en compilations (désormais classificatoires) imprimées, et au dernier prophète royal qu'est Thomas Martin. Ce prophétisme se prolonge après 1830 dans un messianisme, voire un millénarisme, qui exploite les nouvelles révélations (dont les apparitions mariales de La Salette en 1846 dans le cas de Richemont) et identifie les prétendants au Grand Monarque, personnage eschatologique associé au Grand Pape, hérité d'une longue histoire remontant à la fin de l'Antiquité, revivifié durant les années 1820-1840 par la diffusion de recueils de prophéties ${ }^{27}$. Naundorff lui-même prend la place de Martin une fois celui-ci mort, réécrit les Évangiles et fonde une Église; Richemont est identifié au Christ par un

26. Emmanuel de Waresquiel, "Talleyrand et la légitimité...», art. cité. Pour comparer avec d'autres contre-révolutionnaires Gérard Gengembre, La Contre-révolution ou l'histoire désespérante, Paris, Imago, 1989.

27. Exemples d'utilisation des prophéties : dans La Voix d'un proscrit [mensuel naundorffiste], «Extrait de la prophétie dite d'Orval», "Fragment de la prédiction d'une religieuse de Bellay", "Notice sur Martin. - Troisième partie», 20 octobre 1839, p. 250-253, 253-254 et 254-260; «L'an 1840. (Extrait de la Quotidienne du 11 novembre) », 20 novembre 1839, p. 285-287; A. G., «Réponse», «Note sur la prophétie d'Orval ", «Extrait d'un recueil de prédictions, publié par le libraire Bricon, en novembre 1830 ", 20 décembre 1839, p. 298-309, 321-323 et 323-324; A. Gozzoli, "Lettre du Prince au rédacteur", "Notre tâche est remplie : nous allons laisser parler l'avenir", 20 avril 1840, p. 436-441 et 441-444; La vérité sur le fils de Louis XVI connu sous le nom de l'ex-baron de Richemont, Grenoble, Baratier, 1849.

Sur les liens entre survivantisme et prophéties : Philippe Boutry, "Carisma di crisi...", art. cité; Philippe Boutry et Jacques Nassif, Martin l'archange, Paris, Gallimard, 1985; Claude Guillet, La rumeur de Dieu. Apparitions, prophéties et miracles sous la Restauration, Paris, Imago, 1994; Michel Denis, Les royalistes..., ouv. cité, p. 216-218; pour le cas de la Salette, Jean Stern, La Salette. Documents authentiques, 3 tomes, tome 3, 1 er mai 1849 - 4 novembre 1854, Paris, Les Éditions du Cerf, 1991, p. 228, 330, et Michel Corteville, La "Grande Nouvelle» des Bergers de La Salette. Le plus grand amour, les plus fortes expressions, I. L'apparition et ses secrets, Supplément au périodique L'Impartial, $\mathrm{n}^{\circ} 178,2^{\mathrm{e}}$ trimestre 2001, p. 188,202 et 401.

Sur les prophéties dans la France du XIX siècle, Thomas A. Kselman, Miracles and Propheties in Nineteenth-Century France, New Brunswick, Rutgers University Press, 1984; Éloïse Mozzani, Magie et superstitions de la fin de l'Ancien Régime à la Restauration, Paris, Robert Laffont, 1988, p. 250-273, 315330 et 379-382; Jacques Halbronn, Le texte prophétique en France. Formation et forme, thèse d'État sous la direction de Jean Céard, Université Paris 10 -Nanterre, 1999, fo 765-864; Paul Airiau, «Le Grand Monarque dans le catholicisme français (XIXe-XXe siècles)", Le souverain caché-Politica hermetica, $\mathrm{n}^{\circ}$ 14, 2000, p. 66-95 et «Prophétisme politique (dans la France contemporaine)» dans Patrick Sbalchiero [dir.], Dictionnaire des miracles et de l'extraordinaire chrétiens, Paris, Fayard, 2002, p. 645-646; pour une période postérieure, Hilaire Multon, "Les temps sont proches». Prophétisme politique et culture apocalyptique dans le catholicisme français et italien (1859-1878), thèse d'histoire sous la direction de Philippe Boutry, Université Paris 12 - Créteil Val de Marne, 2002. 
de ses partisans; et tous deux doivent faire advenir les temps nouveaux de la régénération sociale voulue par Dieu ${ }^{28}$. La légitimité dépend ici strictement de la conformation à l'ordre divin. Louis-Philippe l'ayant abandonné, elle peut être exploitée.

Mais le prophétisme peut simplement servir à garantir la survie de Louis XVII, à dévoiler l'usurpation (chez Naundorff essentiellement). La primauté de la règle successorale devient alors presque une règle divine. $\mathrm{La}$ rupture est nette par rapport à l'Ancien régime ou aux tenants du droit divin, où Dieu justifie d'abord le pouvoir, avant ses règles de transmission. La légitimité demeure purement ainsi formelle. Le prophétisme a aussi des effets inattendus. Chez Naundorff, les Évangiles réécrits justifient le libéralisme : "Ne vous amassez point des trésors sur la terre, et ne vous occupez point des choses politiques; car là où est votre trésor, là est aussi votre cœur." Le prophétisme alimentant l'anticléricalisme débouche sur l'abandon du catholicisme, y compris par deux prêtres le soutenant ${ }^{29}$. Et Richemont achève sa carrière en faisant appel à l'Assemblée nationale de 1848 pour voir reconnâ̂tre ses droits ${ }^{30}$. S'opère donc une double dynamique pour la légitimité dans l'ultracisme prophétique : sécularisation (par la réduction de la légitimité à une formalité) et recharge sacrale (par le fondement de la légitimité sur l'intervention divine ou la conformation à la volonté céleste), la seconde pouvant paradoxalement alimenter la première. À ce titre, le survivantisme peut être rapproché des "prophètes romantiques", présentant les mêmes caractéristiques, enracinées dans un joachimisme plus ou moins sécularisé : l'utilisation du christianisme comme solution temporelle aux problèmes

28. Charles-Louis, Duc de Normandie [Naundorff], Doctrine céleste, ou l'Évangile de Notre Seigneur Jésus-Christ dans toute sa pureté primitive, tel quill l'a prêché lui-même pendant sa carrière terrestre; Révélé de nouveau par trois Anges du Seigneur, et confirmé par Jésus-Christ lui-même, par la réprobation de la Papauté romaine; avec toutes les preuves de son imposture contre la doctrine de notre Sauveur, s.l., 1839, p. 33.; La Voix d'un proscrit: : De la Doctrine céleste", 20 septembre 1839, p. 216, "Suite de la correspondance», 20 décembre 1839, p. 319, «Plaidoyer de M. Gruau de la Barre pour la Doctrine céleste. - Physionomie des débats», 20 janvier 1839, p. 346; L. Esp. J. V. Claravali del Curso [abbé Louis Veyron], Vie de Mgr le duc de Normandie, fils de Louis XVI et de Marie-Antoinette, roi et reine de France, Que la révolution fit orphelin en 1793, et qu'elle raya du nombre des vivants en 1795, connu dans le monde sous le nom d'ex-baron de Richemont, Paris, Lyon, Imprimerie de Dumoulin et Ronet, 1850; C. [laude] F.[rançois] Nicod, L'avenir prochain de la France entrevu dans les vrais principes de la société, de la liberté, de la souveraineté soit populaire, soit nationale, et dans la révolution de 1789. Ouvrage philosophique, politique et religieux, Paris, Gaume frères, Lyon, Dumoulin et Ronet, 1850 et, sur ce cas, notice par Fernand Rude dans Jean-Marie Mayeur et Yves-Marie Hilaire [dir.], Dictionnaire du monde religieux..., ouv. cité, tome 6, Xavier de Montclos [dir.], Lyon. Le Lyonnais, le Beaujolais, Paris, Beauchesne, 1994, p. 316-318. Sur la pensée religieuse de Naundorff, Paul Airiau, "De Swedenborg à Naundorff : le cas de Melchisédech", Melchisedech-Politica hermetica, ${ }^{\circ}$ 19, 2005, p. 72-82, et notice dans Jean-Pierre Chantin [dir.], Les marges..., ouv. cité, p. 184-187.

29. Charles-Louis, Duc de Normandie, Doctrine..., ouv. cité, p. 11. Cette position déjà présente dans une lettre à M ${ }^{\text {gr }}$ Blanquart de Bailleul, 20/09/1836 (Archives du diocèse de Versailles, dossier Appert). Sur les abbés Laprade et Appert, notices de Paul Airiau, dans Jean-Pierre Chantin [dir.], Les marges..., ouv. cité, p. 6-7 et 155-156. Sur l'évolution anticléricale à partir du prophétisme, Hilaire Multon, «Les temps sont proches"..., ouv. cité.

30. Charles-Louis de Normandie, Adresse aux représentants de la Convention, 25 mai 1848, Paris, Lacour, s.d. 
socio-politiques aboutit à une sécularisation à partir d'une réactivation de la dimension religieuse ${ }^{31}$.

De telles évolutions ne sont pas sans effets sur la théorie des deux corps $\mathrm{du}$ roi. Depuis ses origines, cette fiction juridique peut renforcer le pouvoir royal, par investissement; et le restreindre ou le relativiser, par la distinction entre le corps politique et le roi régnant, ouvrant la voie à une possible autre localisation de la souveraineté qu'en un corps physique. Or, la Révolution, en identifiant le corps politique à la nation, dissocie absolument le corps physique du roi du corps politique, canonisant une distinction gommée par Louis XIV et progressivement réaffirmée au XVIII siècle avec la querelle janséniste et les revendications parlementaires. En même temps, elle finit par identifier radicalement le corps physique de Louis XVI à la monarchie comme forme politique, et peut ainsi supprimer celle-ci en discréditant, dévalorisant et décapitant celui-là, dans une œuvre de donatisme politique déjà pratiqué par la Ligue au XVI siècle $^{32}$.

Les faux dauphins, en tendant à réduire leur projet politique à une règle successorale, minent radicalement la théorie politique de la monarchie. Point de transmission immédiate de la continuité de l'État, aucun exercice de la puissance temporelle, nulle autorité ou majesté. Ne demeure qu'une vacuité : celle du père, que le fils doit combler. Cette extrême simplicité peut être comprise comme l'impossibilité de faire face à la recharge symbolique des deux corps du roi, partiellement efficace au prix d'évolutions, que produit la Restauration ${ }^{33}$. Elle dirait ainsi la réussite du projet restaurateur, et ses limites, puisque la revendication survivantiste ne disparait pas. Elle souligne aussi que le pouvoir se légitime également par son simple exercice, lequel

31. L'abbé calabrais Joachim de Flore (1130-1202), dans ses interprétations de l'Écriture, et plus spécialement de l'Apocalypse, annonçait un âge de l'Esprit à venir rapidement, animé par des hommes spirituels. Son héritage, appelé joachimisme, irrigue largement l'Occident chrétien sous des formes diverses. Henri de Lubac, La postérité spirituelle de Joachim de Flore, 2 tomes, tome 2, De Saint-Simon à nos jours, Paris/Namur, Lethielleux, 1981; Paul Bénichou, Le temps des prophètes. Doctrines de l'àge romantique, Paris, Gallimard, 1977; Bernard Plongeron, «Le christianisme comme messianisme social», dans JeanMarie Mayeur, Charles et Luce Pietri, André Vauchez et Marc Vénard [dir.], Histoire du christianisme, 14 tomes, tome 10, Bernard Plongeron [dir.], Les défis de la modernité (1750-1840), Paris, Desclée, 1997, p. 837-905; Nicole Edelman, Voyantes, guérisseuses et visionnaires en France, 1785-1914, Paris, Albin Michel, 1995; Paul Airiau, "Les millénarismes» dans Jean-Pierre Chantin [dir.], Les marges..., ouv. cité, p. XXVIII-XXXV; Hilaire Multon, «Eschatologie, visions du futur et prophétisme (XIX ${ }^{\mathrm{e}}-\mathrm{XX}{ }^{\mathrm{e}}$ siècles). Essai d'historiographie» dans André Vauchez [dir.], L'Attente des temps nouveaux. Eschatologie, millénarismes et visions du futur, Turnhout, Brepols, 2002, p. 75-95.

32. Alain Boureau, Le simple corps du roi. L'impossible sacralité des souverains français, XVE-XVIII siècles,

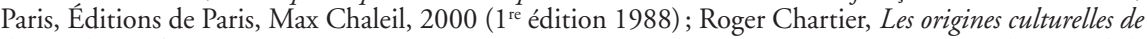
la Révolution française, Paris, Le Seuil, 1990, p. 138-166; Dale K. Van Kley, Les origines religieuses de la Révolution française, 1560-1791, Paris, Le Seuil, 2002, p. 55-56, 83-84, 206-208 et 273-287; Michael Walzer, Régicide et révolution. Le procès de Louis XVI. Discours et controverses, Paris, Payot, 1989; Lynn Hunt, Le roman familial..., ouv. cité, p. 35-38 et 59-68; Annie Duprat, Le roi décapité. Essai sur les imaginaires politiques, Paris, Les Éditions du Cerf, 1992, notamment ch. 3; Antoine de Baecque, Le corps de l'histoire. Métaphores et politique, 1779-1800, Paris, Calmann-Lévy, 1993; Joan B. Landes, Visualizing the Nation. Gender, Representation, and Revolution in Eighteenth-Century France, Ithaca, London, Cornell University Press, 2001, p. 57-80.

33. Emmanuel Fureix, La France des larmes..., ouv. cité, p. 223-272. 
permet le déploiement de codes symboliques renforçant à leur tour ce pouvoir. Elle dit enfin que, d'une certaine manière, chez les faux Louis XVII, la règle d'une monarchie successive, établie comme transcendante, se substitue au corps politique, joue contre le corps physique trônant et en faveur du corps physique revendiquant. Les survivantistes ignorent le corps politique, ne se soucient que du corps mortel. En même temps, ce simplisme est associé de manière complexe à une forte idéologisation. Mais les règles destinées à garantir la continuité de l'État, la perpétuation de la souveraineté par la succession des corps physiques des monarques, la sécurité politique par la stabilité, peuvent-elles être investies d'une participation à une mission du pouvoir? Ou peut-on leur ajouter un projet idéologique qui deviendrait une nouvelle loi fondamentale? La primauté de l'idéologie rend la théorie dépendante de l'application du projet, et lui fait courir le risque de disparaître si d'autres idéologies s'imposent. Mais les survivantistes ne sont pas seuls dans cette évolution. Lors de la mort de Louis XVIII, la Restauration idéologise partiellement les corps royaux : Charles $\mathrm{X}$ s'engage à poursuivre son frère (corps physique) en régnant selon la Charte (corps politique) ${ }^{34}$.

Ainsi, la multiplication des corps physiques avec la réitération des prétendants, la concurrence des incarnations et l'inlassable interrogation sur le possible survivant traduisent, notamment dans l'ultracisme, non seulement qu'on ne peut plus rabattre définitivement le corps politique sur le corps physique, mais que même la théorie des deux corps du roi est mourante, ou morte. Car le corps politique est désormais celui de la nation, ou du peuple, avec laquelle le corps du roi ne peut plus faire un - ou tout au moins, ils doivent composer pour former un corps politique bancal. Les ultras eux-mêmes, en partiels héritiers du libéralisme aristocratique et du jansénisme judiciaire d'Ancien Régime, le savent bien. Abandonnant de fait tout espoir de revenir à une politique fondée sur le roi seul, ils cautionnent, voire revendiquent et organisent contre le roi et ses ministres ce qui s'expérimente comme une forme de parlementarisme. Ils assument leur idéologisation du corps politique sans en tirer toutes les conséquences. L'échec systématique des Louis XVII souligne également que la naturalité du corps ne peut efficacement s'opposer à l'artificialité du contrat social. Le corps royal n'est plus une politique, quoi qu'ils en veuillent. Ils expérimentent le même échec que Charles X se faisant sacrer, tentant d'investir divinement de la légitimité son corps comme corps royal et par là la monarchie restaurée. Mais ce souci differe trop de celui de la majorité des responsables politiques, qui veulent achever l'histoire de la France comme sujet corporellement distinct du roi, à la manière de Rémusat en 1840, qui montre la continuité physique de la nation en installant à la Chambre des pairs les statues d'illustres Français des siècles précédents dont des descendants sont pairs ${ }^{35}$.

34. Idem, p. 236.

35. Pierre Karila-Cohen, "Charles de Rémusat... », art. cité, p. 414. 
Ignorant en fait la théorie des deux corps du roi mais alimentant une incertitude sur le détenteur du pouvoir, les survivantistes, paradoxalement, participent à la désincarnation politique du libéralisme. Ainsi, à rebours, montrent-ils que la Révolution l'a emporté. La souveraineté de la nation est devenue indépassable. Comme l'écrit Le Siècle à propos du défi naundorffiste aux légitimistes venus visiter le duc de Bordeaux à Londres en décembre 1843 : «Pauvre légitimité! S’il faut en effet la chercher dans la personne des princes et non dans la volonté des nations, Dieu aurait bien dû la marquer sur leur front d'un signe ineffaçable. ${ }^{36}$.

Les multiples Louis XVII et l'impossibilité de les faire disparaître montrent combien les Français sont agités par une profonde indécision politique, combien la légitimité change de sens et combien se renouvellent ou se décomposent les justifications de son fondement, y compris chez les ultras. Se déroulent alors des itinéraires politiques complexes. Modeste Gruau, d'origine légitimiste, débute ainsi une carrière judiciaire interrompue en 1830. En lien avec un milieu prophétique, il reconnaît en Naundorff Louis XVII, puis accepte ses revendications messianiques. Il s'oriente alors vers un libéralisme religieux débouchant dans un libéralisme politique et une hostilité radicale au monde légitimiste et au catholicisme. Sur ces bases contestataires, se construisent et se perpétuent également de petits milieux. La petite fille de Rambaud épouse Léon Verger, un neveu du secrétaire d'Étienne de Joly, éphémère ministre de la Justice en juillet-août 1792 et qui reconnut en Naundorff Louis XVII; une des nièces de la même Rambaud épouse Xavier Laprade; une autre épouse Augustin Solard, dont le frère, Auguste, bonapartiste marié à Églantine Pégot (fille du général d'empire Jean Pégot et belle-fille du littérateur Emile Marco de Saint-Hilaire, fils de Denis Marco), finit secrétaire général de la préfecture d'Orange sous le Second Empire ${ }^{37}$. Les faux Louis XVII peuvent ainsi servir diachroniquement et synchroniquement à apprécier quasiment de manière micro-historique les voies de la politisation, l'articulation des héritages et des projets politiques et les évolutions des opinions politiques des Français.

Paul Airiau est docteur en histoire (IEP de Paris) et professeur agrégé d'histoire dans l'académie de Paris

36. Le Siècle, 9 décembre 1843, [p. 3].

37. Paul Airiau, notice Gruau dans Jean-Pierre Chantin [dir.], Les marges..., ouv. cité, p. 116-117; Guy de Rambaud, Pour l'amour..., ouv. cité; Arch. nat. F1 B1 173/18, «Solard Auguste». 